\title{
Die RÄUMLICHe SPRACHe DeR ERFAHRUNG. DIE INNERE ZEIT UND DER INNERE RAUM
}

\author{
EL LENGUAJE ESPACIAL DE LA EXPERIENCIA. \\ EL TIEMPO INTERIOR Y EL ESPACIO INTERIOR
}

\author{
Victor Molchanov \\ Russische Staatsuniversität für Geisteswissenschaften, Moskau \\ victor.molchanov@googlemail.com
}

\begin{abstract}
This investigation addresses the internal experience as a spatial phenomenon. Ascertaining the difference between internal and external experience as a space metaphor leads to the question of the source of the space metaphors in principle. The analogy between time and space and the space metaphors in Husserl's conception of time are considered. The question of the temporality of consciousness, evidence, and internal experience are brought to the fore by comparing Brentano's and Husserl's conceptions. The difference between the direct and indirect modes of consciousness by Brentano, his conception of time as the boundary in the continuum, and his concept of the proteraesthesis proves to be relevant for the concept of internal experience as a spatial phenomenon. The essay calls in question the self-sufficiency of time-experience and the traditional correspondence of time to the internal experience and of space to the external one. The relation of the Here/There- and Now/Thenexperience is discussed. I intend to elucidate the experience of boundary as a primary internal experience from which the space-experience arises. In its turn the boundary-consciousness has its origin in the differentiation-experience. In the latter there is no time, the internal one included, but there is a boundary-experience as a primary and indirect experience. Time is $\mathrm{s}$ shadow of space; it originates from the manifold of human spaces.
\end{abstract}

Keywords: Experience. Time. Space. Boundary. Consciousness. Internal. Husserl. Brentano.
Resumen: Esta investigación aborda la experiencia interna como un fenómeno espacial. Determinar la diferencia entre la experiencia interna y externa como una metáfora del espacio conduce a la cuestión de la fuente de las, en principio, metáforas espaciales. Aquí se atiende la analogía entre el tiempo y el espacio y las metáforas espaciales en la concepción del tiempo de Husserl. Se ponen de relieve la cuestión de la temporalidad de la conciencia, la evidencia y la experiencia interna, comparando las concepciones de Brentano y Husserl. La diferencia entre los modos de conciencia directos e indirectos de Brentano, su concepción del tiempo como el límite en el continuo, y su concepto de la proteótesis, son relevantes para el concepto de experiencia interna como un fenómeno espacial. El ensayo cuestiona la autosuficiencia de la experiencia del tiempo y la correspondencia tradicional del tiempo con la experiencia interna y del espacio con la externa. Se discute la relación de la experiencia Aquí/Ahí y de la experiencia Ahora/Luego. Mi intención es esclarecer la experiencia de la frontera como una experiencia interna primaria de la cual surge la experiencia espacial. A su vez, la conciencia de frontera tiene su origen en la experiencia de diferenciación. En esta último no hay tiempo -incluso el interno-, pero hay una experiencia límite como experiencia primaria e indirecta. El tiempo es la sombra del espacio; se origina en la multiplicidad de espacios humanos.

Palabras clave: Experiencia. Tiempo. Espacio. Límite. Conciencia. Interno. Husserl. Brentano. 
Die Differenz zwischen dem Inneren und dem Äußeren ist eine grundlegende in der menschlichen Welt: von pränataler Existenz und Geburt bis zum Tode und Beerdigung. Die Differenz zwischen der inneren und äußeren Erfahrung ist allgemeingültig in den Geisteswissenschaften, zunächst in Psychologie und Philosophie. Von besonderer Bedeutung wird diese Differenz wie bekannt in der Phänomenologie Brentanos und Husserls.

Auch der Common sense kennt diese Differenz, und die Ausdrücke wie "innere Erfahrung" und "äußere Erfahrung" wurden bis zu solchem Grade verbreitet, dass man ihren räumlichen Charakter übersieht. Man kann diese Ausdrücke für Metaphern halten, und in einem gewissen Sinn mit Recht, aber das beantwortet nicht die Frage: warum gerade räumliche Metaphern für die Beschreibung dieser Erfahrungsdifferenz gebraucht werden. Gibt es überhaupt einen anderen Ursprung metaphorischer Ausdrücke als die Räumlichkeit der Dinge, der Situationen, der Ereignisse usw? "Ur-sprung" und "Meta-pher" beziehen sich auch auf das Räumliche, das im Grunde genommen eine Quelle aller Metaphorik ist.

Was ist der Grund des Unterschieds zwischen innerer und äußerer Erfahrung? Die Antwort, dass dies der Leib ist, sogar der eigene Leib, führt auch zu räumliche Bestimmungen.

Traditionell bringt man Raum in Verbindung mit der äußeren Erfahrung, Zeit aber mit der inneren. Zumindest herrscht diese Betrachtungsweise vor: anfangend mit Kant bis zu Bergson, Husserl, und darüber hinaus. Dabei ist die Lehre Brentanos bemerkenswert: psychische Phänomene haben weder räumliche noch zeitliche Bestimmungen. Wenn sie weder temporal noch räumlich sind, sind sie vielleicht ewig? Von welchem Standpunkt aus kann man aber einen Zugang zu einer solchen Ewigkeit bekommen?

Die räumliche Sprache stellt die erwähnten Korrelationen (der Zeit entspricht das Innere und dem Raum das Äußere) in Frage. Sie ist gebräuchlich nicht nur für die Beschreibung des Inneren im Allgemeinen, zum Beispiel für die des Bewusstseins als Reflexion oder als Richtung auf Gegenstände, sondern auch für die Beschreibung der Zeit, zum Beispiel, als kurz oder lang. Die Grundworte, die den Grundraum menschlicher Erfahrung zum Ausdruck bringen - gehen, kommen, stehen, bringen und ihre Ableitungen - werden auch sehr oft für die Beschreibung der Zeit gebraucht. Selbst das Wort "Erfahrung" ist von dem Verb "fahren" abgeleitet, und seinem Ursprung nach ist räumlich. 
Nun stellt sich die Frage: hat die Zeit ihre eigene Sprache oder ist die zeitliche Sprache eine sekundäre, aus räumlicher Sprache erwachsene?

Es handelt sich dabei nicht um sekundäre Zeitbestimmungen, sondern um die grundlegende Reihe: Gegen-wart, Ver-gangenheit, Zu-kunft - alle diese Wörter beziehen sich letzten Endes auf das Räumliche, i.e., auf die Sphäre der Bewegung, der Wendung, des Stehens. Auch die andere Bestimmungsreihe der Zeit - früh und spät, früher und später tragen die Spuren des Räumlichen, aber fast unbemerkbar ${ }^{1}$. Auf die Unselbständigkeit der Zeitsprache verweist aber nicht nur die Anwesenheit der räumlich beziehenden Formen in entsprechenden Wörtern. Vielmehr spricht darüber die Tatsache, dass alle Zeitbestimmungen letzten Endes Raumbestimmungen sind. Kaum kann man daran zweifeln, wenn die Rede von der objektiven Zeit ist. Minuten, Stunden und andere objektive Bestimmungen, sowie früher und später, gehen - objektiv verstanden - bekanntlich auf die Erdebewegung zurück. Wie steht es mit innerer Zeit und mit innerem Raum? In welchem Sinne kann man aber über den inneren Raum sprechen? Vielleicht könnte uns den Weg zu diesem Raum die Zeitphänomenologie zeigen?

Nicht nur in der Umgangssprache, sondern auch in den philosophischen Lehren begegnen wir Analogien zwischen Raum und Zeit, sowie die räumliche Beschreibung der Zeit. Selbst ein von Bergson geprägter anti-räumlicher Begriff "elan vital" bezieht sich im Sinne Sprung oder Schwung auf den Raum. Viele räumliche Metaphern kann man in Husserls Phänomenologie des inneren Zeitbewusstseins finden. Zu Beginn der Vorlesungen vom Jahre 1905, in §1, der den Titel "Ausschaltung der objektiven Zeit" trägt, stellt Husserl die objektive Zeit der immanenten Zeit des Bewusstseinsverlaufes gegenüber. Aber die Explikation des letzteren fängt nicht mit einer Deskription an; sie beginnt mit der Analogie zwischen "ursprünglichem Zeitfeld" und dem Gesichtsfeld. Kaum ist es nötig zu verweisen, dass das Wort "Feld" seinen Ursprung aus räumlichen Beziehungen nimmt. Also beginnt die Ausschaltung der objektiven Zeit mit der Ausschaltung des objektiven Raums. Wenn das Gesichtsfeld keine messbaren Distanzen aufweist, soll auch die innere Zeit keine messbaren Intervalle in sich enthalten. Aber gibt es die Zeit ohne Zeiträume?

\footnotetext{
${ }^{1}$ Das ausreichende Material kann man in dieser Hinsicht in verschiedenen etymologischen Wörterbüchern finden. Ein gutes Beispiel ursprünglicher Räumlichkeit der zeitlichen Bedeutung gibt uns das englische Wort "always": alle Wege; das deutsche Wort "Bewegung" verweist auch auf einen Weg.
} 
Bei der Charakterisierung der Wahrnehmung von Dauer wendet sich Husserl wieder an die Analogie zum Raum, diesmal zum objektiven:

Die Punkte der Zeitdauer entfernen sich für mein Bewusstsein analog, wie sich die Punkte des ruhenden Gegenstands im Raum für mein Bewusstsein entfernen, wenn ich "mich" vom Gegenstand entferne. Der Gegenstand behält seinen Ort, ebenso behält der Ton seine Zeit, jeder Zeitpunkt ist unverrückt, aber er entflieht in Bewusstseinsfernen, der Abstand vom erzeugenden Jetzt wird immer größer. ${ }^{2}$

Die wichtigsten von Husserl in Bezug auf Zeit angewandten räumlichen Metaphern unterscheiden sich nicht im Allgemeinen von denen, die weit verbreitet sind: versinken, verlaufen, übergehen, usw. In der Regel stehen sie mit der Bewegung und den räumlichen Verhältnissen in Verbindung. Was die spezifischen Termini der Zeitphänomenologie Husserls betrifft - Jetzt-punkt, Retention und Protention - beziehen sie sich auch auf den Raum. Der "Punkt", sei es eine geometrische oder kartografische Idealisierung, ist eine räumliche Bestimmung. Die Übertragung einer solchen idealisierten räumlichen Bestimmung auf die Zeit bringt als Folge einen zeitlichen Punkt. Unter den verschiedenen räumlichen und zeitlichen Punkten gibt es die beiden, zumindest in Husserls Phänomenologie wichtigsten: Hier-Punkt und Jetzt-Punkt. "Hic et nunc" wurde zu einem der Schlagworte der Phänomenologie. Ist das nicht eine Tautologie?

Dass Hier und Jetzt verschieden sind, akzeptiert man ohne weiteres. Doch ist die Frage dieser Differenz diskutierbar. Es handelt sich freilich nicht um die Differenz der idealisierten Hier -und Jetztpunkte-, sondern um die Erfahrungsweisen, wo jedes Hier immer ein Jetzt, und jedes Jetzt immer ein Hier ist.

Das alte Sprichwort: "Hic Rhodos, hic salta" kann uns Hilfe für die Analyse leisten. Es bedeutet "Springe Hier und nicht Dort" oder "Springe Jetzt und nicht Dann". Es scheint ein großer Unterschied zwischen "Dort" und "Dann" zu sein. Es ist offensichtlich, dass "dann" zu springen unmöglich ist, weil "dann" bereits in der Vergangenheit ist. Ganz im Gegenteil scheint es möglich zu sein, "dort" zu springen, nämlich in einem Rhodos, das nicht in der Vergangenheit ist, und man braucht nur ein Zurück zu Rhodos für einen solchen Sprung. Aber für jemanden, der sich nicht in Rhodos befindet, ist es unmöglich in Rhodos zu springen. Rhodos

2 E. Husserl, Zur Phänomenologie des inneren Zeitbewusstseins, hrsg. von Rudolf Boehm, (Husserliana X), Dordrecht: Martinus Nijhoff, 1966, S. 25. 
als ein geografischer Ort setzt fort zu existieren, aber der Raum dieses alten Sprungs ist unwiderruflich verloren. Der verlorene Raum ist gleich der verlorenen Zeit. Vielmehr vergehen nicht die menschlichen Zeiten, sondern die menschlichen Räume, und die Zeit ist nur eine gegenseitige Korrelation von Räumen.

Ist aber die Zeit etwas anderes als ein Mittel der Messung? Der Sprungraum in Rhodos ist lange vorbei, zusammen mit der Erdebewegung. Der Raum des geforderten Sprungs, der hier sein sollte und nicht in Rhodos (nehmen wir an, dass das gerade wir sind, die den Sprung hier forderten), ist der Raum der Gegenwart, er kann nicht nur Hier-Raum, sondern auch Jetzt-Raum genannt werden.

Brentano hat eine andere Sicht. Für inn spielt der Unterschied zwischen "Hier" und "Jetzt" eine besondere Rolle:

Der Ortpunkt des Hier ist nicht identisch mit dem Zeitpunkt des Jetzt und der eines Dort mit dem Zeitpunkt eines Dann, wenn auch dasselbe hier und jetzt ist und dort und dann. Der Ortpunkt ist Grenze für ein Räumliches in vielen, ja vielleicht in allen denkbaren räumlichen Richtungen, aber niemals Grenze in einer der beiden zeitlichen Richtungen, und vom Zeitpunkt gilt das Umgekehrte. ${ }^{3}$

Brentano macht diese Unterscheidung bei seiner Kritik an den physikalischen Begriff der vier-dimensionalen Raum-Zeit, wo Raum und Zeit ihre Selbstständigkeit verlieren. Aber unabhängig von dieser Kritik ist es offensichtlich, dass Raum und Zeit für Brentano die verschiedenen Wesen sind, die in der Erfahrung eine unterschiedliche Rolle spielen. Dennoch ist eine dritte Position möglich, die ich hier (und jetzt) entwickeln möchte, nämlich: Zeit hat nur relative Selbstständigkeit und letzten Endes ist zum Raum reduzierbar.

Was meint Brentano, wenn er schreibt "dasselbe hier und jetzt und dort und dann"? Es kann nur bedeuten, dass alle diese Punkte sich auf das gleiche Ereignis beziehen. Aber könnte es sein, dass ein Hier-Ereignis nicht ein Jetzt-Ereignis wäre? Die negative Antwort ergibt sich aus einer Erfahrung der Beteiligung an einem Ereignis, die positive von einem objektiven Gesichtspunkt aus.

Stellen wir die Frage anders: Ist es möglich für Jetzt-Ereignis, nicht Hier stattzufinden? Dabei sind auch zwei gegensätzliche Antworten möglich. Wenn wir "Jetzt" als einen Punkt oder Zeitraum (dieser Ausdruck spricht für sich selbst) S. 208

${ }^{3}$ F. Brentano, Philosophische Untersuchungen zu Zeit, Raum, Kontinuum, Hamburg: Meiner, 1976, 
interpretieren, der durch ein Chronometer bestimmt ist, und "Hier" als eine physische oder kartographische Charakteristik, wäre die Antwort positiv. In der Tat kann es verschiedene Nicht-Hier-Ereignisse geben, die in einem bestimmten Moment vorkommen. Außerdem kann man sagen, dass viele Ereignisse "Hier" aber nicht "Jetzt" bereits geschehen seien und in der Zukunft geschehen würden. Aber innerhalb des Ereignisses sind für uns "Hier" und "Jetzt" untrennbar: es kann nicht ein "Jetzt-Moment" ein "Nicht-Hier-Moment" sein und umgekehrt.

Die Untrennbarkeit von Hier und Jetzt bedeutet nicht ihre Symmetrie. "Stattfinden" (take place) ist der erste Charakterzug des Ereignisses, der gestattet einer Stätte oder Stelle oder einem Ort ziemlich unbestimmt zu sein, und im gegebenen Moment zu sein ist von sekundärer Bedeutung, auch wenn der Moment streng festgelegt werden könnte.

Jedes Ereignis ist in erster Linie eine Konstellation und Korrelation verschiedener Orte und Räume, ihre gegenseitige Korrespondenz und Divergenz, Durchdringen und Umstrukturierung. Insbesondere betrifft dies "innere Ereignisse", d.h. Erlebnisse. Unser Wahrnehmen, Denken oder Gedächtnis vollzieht sich in einer Bedeutsamkeits- und Präferenzhierarchie der Lebenswelt. In einer solchen Hierarchie gibt es auch einen Unterschied zwischen "Hier" und "Dort", d.h. dem Vorder - und Hintergrund -, was mit dem Unterschied zwischen "Jetzt" und "Dann" zusammenfällt.

Nicht innere Zeit, sondern vielmehr innerer Raum strukturiert unsere innere und äußere Erfahrung. Erlebnisse, wie immer man sie interpretieren könnte - als Actcharaktere oder als Unterscheidungsleistungen - verschwinden nicht in dem Bewusstseinsstrom, vielmehr werden sie in einer Hierarchie des inneren Raums umstrukturiert. Der einfachste Fall dieser Umstrukturierung ist ihr Rückzug in den Hintergrund und ihr Treten in den Vordergrund.

Die Trennung von "Hier" und "Jetzt" bei Brentano ergibt sich aus seiner mathematischen Idealisierung von Raum und Zeit. Der Raum ist konzipiert als eine Mannigfaltigkeit von Punkten in einem Kontinuum, in dem jeder Punkt eine Grenze unendlicher Menge von Richtungen ist. Die Zeit ist ein Kontinuum von Punkten der geraden Linie, wo die Gegenwart die Grenze zwischen Vergangenheit und Zukunft ist. Also trennt Brentano Raum und Zeit unter einem quantitativen und quasi-geometrischen Gesichtspunkt. Dennoch möchte ich die wichtigen Momente in dieser Konzeption hervorheben, die indirekt gegen eine solche Trennung sprechen. 
Erstens sind Punkte mit Grenzen identifiziert. Zweitens ist die Grenze ein Hauptmerkmal sowohl von Ort (Raum) als auch von Zeit. Drittens kann der Punkt im Raum beliebig gewählt werden, aber der Gegenwartspunkt erweist sich nur als die Gegenwart und er kann nicht willkürlich und abstrakt herangezogen werden. Er steht immer mit der Hier-Erfahrung, die immer konkret ist, in Verbindung. Sollte dies nicht zeigen, dass Brentanos Konzeption der Zeit einen Schritt in die Richtung beim Suchen nach dem primären Erfahrungsraum darstellt?

In Grunde genommen ist die Grenze ein wesentliches Merkmal des Raums, das sich sowohl auf einen objektiven oder lebensweltlichen Raum, als auch auf den Raum des Erlebens beziehen kann. Es scheint dabei die Gegenwart als Grenze, die räumliche Metapher zu sein. Aber woher kommt diese Metapher? Was für eine Quelle hat die Metapher "Grenze" und die Metaphern überhaupt?

Die Hauptquelle aller Metaphern sind Leib und Raum, genauer gesagt, die Differenz zwischen innen. Was die Deskription betrifft, reduziert sich sowohl die objektive Zeit, als auch die Zeiterfahrung und innere Erfahrung überhaupt auf die leiblich räumliche Beschreibung. Seinerseits aber sind Nähe und Ferne, Grenze und Weite, recht und links, oben und unten, vor und hinten auf etwas anderes als das Räumliche unreduzierbar. Die Raumsprache ist ursprünglich und selbständig. Die Zeitsprache ist sekundär und abhängig. In diesem Sinne und nicht nur in diesem ist die Zeit von dem Raum abhängig.

Wenn die objektive Zeit sich auf die räumliche Bewegung reduziert (Zeit ist nichts anderes als die Zahl der Bewegung), worauf ist die räumliche Bewegung reduzierbar?

In einem späteren Text Husserls finden wir einen Versuch, die Erde nicht als ein Planet zu erörtern ("er-örtern" ist auch ein räumliches Wort), sondern als einen absoluten Grund jeder Erfahrung. In solchem Fall bewegt sich die Erde nicht und auch ruht sie nicht. In der Tat ist der objektive Raum und die objektive Bewegung eine Abstraktion von der lebensweltlichen Erfahrung. Aber die Frage bleibt immernoch: was für eine Erfahrung liegt der lebensweltlichen Raum - und Bewegungserfahrung - zugrunde? Was gehört zur primären Erfahrung des Raums und zum primären Raum der Erfahrung? 


\section{GRENZE UND INNERE ERFAHRUNG}

In keinem Punkt weichen sich Brentano und Husserl voneinander so stark ab, wie in der Frage der Zeitlichkeit des Bewusstseins. Brentano stellt die Frage über das Bewusstsein der Zeit, aber er erkennt weder den Bewusstseinsstrom noch die innere Zeit an. Das Bewusstsein des Zeitlichen ist nicht in sich selbst das Zeitliche. Im Gegenteil, für Husserl

ist (es) ja evident, dass die Wahrnehmung eines zeitlichen Objektes selbst Zeitlichkeit hat, dass Wahrnehmung der Dauer selbst Dauer der Wahrnehmung voraussetzt, dass die Wahrnehmung einer beliebten Zeitgestalt selbst ihre Zeitgestalt hat. Und sehen wir von allen Transzendenzen ab, so verbleibt der Wahrnehmung nach allen ihren phänomenologischen Konstituentien ihre phänomenologische Zeitlichkeit, die zu ihrem unaufhebbaren Wesen gehört. ${ }^{4}$

Also ist das Bewusstsein "in its deepest home" (L. Brouwer) als zeitlich interpretiert. Und noch mehr: in der V. Logischen Untersuchung versucht Husserl, Brentanos nicht-zeitliche innere Wahrnehmung des Bewusstseinsaktes in die Reflexion auf den Bewusstseinsstrom und seiner Phasen zu verwandeln. Aber diese Transformation hat eine solche Eigentümlichkeit, dass ihre Mittel nichts anderes ist als Retention, d.h. ein Element des Zeitbewusstseins. Sollte also das zeitliche Bewusstsein das Nicht-zeitliche in das Zeitliche verwandeln? Und wird nicht zusammen mit der zeitlichen Dehnbarkeit des inneren Bewusstseins auch die Evidenz selber dehnbar.

Wie wir gesehen haben, gibt es zu viel Raum in dieser Husserlschen Zeit. Der Verdacht entsteht, dass sich die Raumerfahrung zumindest auf einer und derselben Ebene wie die zeitliche Erfahrung vollziehen sollte. Husserls Beispiele für solche evidenten Zustände, wie "diese Lust, die mich erfüllt; diese Phantasieerscheinung, die mir eben vorschwebt $u$. dgl"5. verweisen vielmehr auf nicht zeitlichen als auf zeitlichen Charakter dieser Zustände. "Erfüllen" und "Schweben" scheinen räumliche Metaphern zu sein. Dies ist der Fall, wenn der gemeinte Raum objektiv ist. Aber diese Metaphern führen uns zur nicht-metaphorischen Ebene

\footnotetext{
${ }^{4}$ E. Husserl, Zur Phänomenologie des inneren Zeitbewusstseins, S. 22.

${ }^{5}$ E. Husserl, Logische Untersuchungen, Bd. II (1), hrsg. von Ursula Panzer, Husserliana XIX (1), Dordrecht: Kluwer, 1984, S. 368.
} 
der Erfahrung, die eine innere und räumliche ist. Husserl selbst hat die grundlegende Unterscheidung zwischen leerer und erfüllter Intention eingeführt, so dass Bewusstsein als eine innere räumliche Unterscheidungsleistung vorgestellt ist. Kaum kann man eine Erfahrung des Temporalen sowohl in der oben genannten Unterscheidung, als auch in den erwähnten Zuständen haben. Die Lust beispielweise kann natürlich dauern, und man kann ihre verschiedenen Phasen in der Erinnerung post factum verfolgen, aber nichts Zeitliches gibt es im Vollzug ihrer Erfahrung. Im Gegenteil, erweist sich die Lust oder die Trauer als ein Bild der Ewigkeit, sie füllt uns ohne Rest und entlässt alles, was man gewöhnlich als Zeitliches bezeichnet: Sorgen, Pläne, Zweifel.

Es gibt eine Einheit von Ich-Erfahrung, Zeiterfahrung und Raumerfahrung in solchen Zuständen wie das Evidenzerleben, Freude, Trauer usw. Aber die tiefste (eine räumliche Metapher!) Erfahrung in einer solchen Einheit ist doch räumlich. Das ist die Erfahrung der Grenzen, Übereinstimmungen und Trennungen. In der Beschreibung von Evidenz bei Husserl, seinen Bemühungen zuwider, gibt es keine zeitliche Charakterisierung, sondern eine räumliche: eine Übereinstimmung der Intention und ihrer Erfüllung. Dies gilt auch von den Begriffen des Phänomens und der Wahrheit, die durchaus räumlich expliziert sind. Besonders deutlich wird dies bei Heidegger, mit solchen "Existenzialen", als Erschlossenheit, "sich selbst zeigen", "Außer sich selbst" usw. Nichtsdestoweniger fehlt solchen Bestimmungen etwas, um die Erfahrungsbeschreibung zu werden: Grenze als eine der wichtigsten Erfahrungsstrukturen kommt nicht in Betracht.

Im Gegensatz zu Husserl versuchte Brentano, weder die Zeit durch räumliche Metaphern aufzuklären, noch das Bewusstsein als zeitliches darzustellen. Zwei Unterscheidungen liegen Brentanos Lehre über die Zeit zugrunde: (1) Diejenige zwischen direktem und indirektem Modus des Bewusstseins, (2) diejenige zwischen der Empfindung und Proto-Empfindung (Proterästhese). Die erste führt zur These von der Bezogenheit aller Vergangenheitsmodi auf die Gegenwart und zur Auslegung der vielfältigen Modi der Vergangenheit als indirekte. Seinerseits führt dies zur Negation absoluter zeitlichen Unterschiede und zum Verständnis der Zeit als Kontinuum, wo die einzige Realität die Gegenwart ist als ein Punkt im Kontinuum und eine Grenze zwischen zwei Reihen indirektrer Modi - der vergangenen und der zukünftigen.

Die zweite Unterscheidung sollte erklären, wie das Bewusstsein der Zeit möglich ist. Die geweckte Empfindung produziert eine andere Empfindung, die 
ihren Ursprung nicht in jenem Objekt hat, das die erste Empfindung hervorrief, sondern in der ersten Empfindung selber. Was sich als Gegenwart in der ersten Empfindung repräsentiert, zeigt sich als jüngste Vergangenheit in der zweiten. Die Proto-Empfindung schiebt also das Objekt der ersten Empfindung in die Vergangenheit. Dann erzeugt die erste Proto-Empfindung die nächste ProtoEmpfindung, die ihr Objekt als ein solches repräsentiert, das sich in Bezug auf seinen vorausgehenden Zustand wieder als soeben vergangenes darstellt. Mit anderen Worten schiebt die zweite Proto-Empfindung ihr Objekt weiter in die Vergangenheit. Und die Fortsetzung dieser Reihe gibt uns den Zeitfluss. Wenn der Ursprung unserer Raumvorstellung in den äußeren Objekten zu finden ist, wie Brentano behauptet, hat unser Zeitbewusstsein einen inneren Ursprung. Aber dies bedeutet nicht, dass die Proto- Empfindung und das Verhältnis zwischen ihr und der ersten erzeugenden Empfindung zeitlich ist. Das Bewusstsein der Zeit geht nicht in die Zeitlichkeit des Bewusstseins über. Brentano hat selbst dies folgenderweise zum Ausdruck gebracht:

Die Evidenz der inneren Wahrnehmung ist also ganz auf die Gegenwart beschränkt. Ja, dies gilt von der inneren Empfindung überhaupt. Sie sagt mir nichts als dass ich jetzt diese Ästhese samt einer kontinuierlichen Proterästhese von physischen Phänomenen habe. Würde sie selbst auch von einer inneren Proterästhese begleitet sein, so ist leicht nachweisbar, dass dies zu einer unendlichen Komplikation führen würde. ${ }^{6}$

Ein weiteres Argument für die Nicht-Zeitlichkeit des Bewusstseins finden wir in Brentanos Unterscheidung zwischen der Wahrnehmung der primären und sekundären Objekte (ein primäres Objekt ist nicht immer ein äußeres). Es gibt keine zeitliche Beziehung zwischen ihnen. Wir möchten hinzufügen: sowie zwischen dem Vorder - und Hintergrund - eines Gesichtsfeldes und jeden Erfahrungsbereiches überhaupt.

Wenn die innere Erfahrung keine zeitlichen Beziehungen in sich enthält, von welcher Art der Beziehungen kann die Rede sein? Welche Art von Beziehung gibt es zwischen der inneren und äußeren Wahrnehmung, zwischen dem direkten und indirekten Modus, zwischen dem Vorder - und Hintergrund - der Wahrnehmung? Streng genommen ist es nicht eine abstrakte Beziehung, sondern die Erfahrung der Grenze, die die innere Erfahrungsstruktur bestimmt. Für Brentano ist die

\footnotetext{
${ }^{6}$ F. Brentano, Vom sinnlichen und noetischen Bewusstsein, Leipzig: Felix Meiner, 1928, S. 51.
} 
Grenze keine Erfahrungscharakteristik. Vielmehr erweist sie sich bei inm als ein ontologisches Wesen, das die zwei anderen ontologischen Wesen - Raum und Zeit - verschieden charakterisiert. Er lehnt nicht nur den zeitlichen, sondern auch den räumlichen Charakter des Bewusstseins und der inneren Erfahrung ab.

Erfahrungsmäßig aber ist die Grenze das erste Element der Räumlichkeit und die tiefste Wirklichkeit der inneren Erfahrung. Das zweite Element einer solchen Räumlichkeit zeigt sich als eine Erfahrung von Weite. Die Unterscheidung zwischen Grenze und Weite liegt einer Erfahrung von Raum und dem Raum der primären Erfahrung zugrunde. Also nicht die Raumvorstellung oder besser gesagt Raumerfahrung produziert die Erfahrung der Grenze und der Weite, sondern im Gegenteil hat die Erfahrung von Raum seinen Ursprung in ihrem Unterschied. Diesem Unterschied, wie allen Unterschieden überhaupt, entspricht eine Unterscheidungsleistung, die in diesem Fall als primäre Raumunterscheidung bezeichnet werden kann.

In aller Unterscheidung gibt es nichts Zeitliches, aber es gibt eine Erfahrung der Grenze, die auch indirekt sein kann, aber dennoch bleibt sie eine Grundlegung jeder Erfahrung. In jeder Unterscheidung realisiert sich die Unterscheidung der Grenze und Weite, sowie auch die der Hierarchie und Erschlossenheit, des Vorder - und Hintergrundes. Letzteres ist besonders wichtig: die Grenze und Weite können ihren Status wechseln; damit sie in den Vordergrund treten und in den Hintergrund zurückweichen, verändern sie den primären Erfahrungsraum.

Wo sollte man nun den Ursprung und die Stelle der Zeit suchen? Wozu überhaupt die Zeit und besonders die innere Zeit, wenn die tiefste Erfahrungsschicht räumlich ist? Die Zeit steht wirklich mit der Zahl und der Mannigfaltigkeit in Verbindung. Wenn der lebensweltliche Raum und die Raumerfahrung ein und einzig wären, dann wäre die Zeit nicht nötig. Doch gibt es notwendigerweise nicht einen, sondern viele Räume und Räumeerfahrungen, die eine "prinzipielle Koordination" erfordern. Diese Funktion nimmt auf sich die Zeit. (Bemerkenswert ist, dass die Zeit bei Kant, Husserl und letzten Endes bei Heidegger als eine Synthesis interpretiert wird). Dabei erhebt in der Regel ein solcher Koordinator den Anspruch, der Ursprung und das Grundprinzip der Erfahrung zu sein. Offenbar erwächst die Zeit aus menschlicher Welt und Erfahrung, aber es bedeutet nicht, dass der erwähnte Anspruch gerechtfertig ist. Wenn die räumliche Metapher in Bezug auf die Zeit als solche und nicht auf ihre "Eigenschaften" (fließt, vergeht, kommt, usw.) angewendet wird, so könnte man sagen: die Zeit ist ein Raum- 
schatten. Der Schatten ist nicht ein Nichts, aber er lebt auf fremde Kosten und die Zeit lebt auf Kosten der Räume und seiner Korrelation. Dies gehört sowohl zu den objektiven und lebensweltlichen Zeiten, als auch zur inneren Zeit, die den inneren Raum verschattet. Die Zugehörigkeit der Raum - und Zeiterfahrung - ist die Quelle unvermeidlicher, unentbehrlicher, und wenn man will, transzendentaler Illusion der Selbständigkeit der Zeit und der Zeiter-fahrung.

\section{LITERATURLISTE}

BRENTANO, Franz. Vom sinnlichen und noetischen Bewusstsein [Psychologie III], Leipzig: Felix Meiner, 1928..

-, Philosophische Untersuchungen zu Raum, Zeit, und Kontinuum, Hamburg: Meiner, 1976

HUSSERL, Edmund. Zur Phänomenologie des inneren Zeitbewusstseins (18931917), hrsg. von Rudolf Boehm (Husserliana X), Dordrecht: Martinus Nijhoff, 1966.

-, Logische Untersuchungen, Bd. II (1), hrsg. von Ursula Panzer. Husserliana XIX (1), Dordrecht: Kluwer, 1984. 